\title{
O Prêmio Nobel de Física de $2014^{+*}$
}

\author{
José Maria Filardo Bassalol \\ Fundação Minerva \\ Belém - PA
}

\section{Resumo}

Neste artigo, trataremos do Prêmio Nobel de Física de 2014, concedido aos físicos: os japoneses Isamu Akasaki (n.1929) e Hiroshi Amano (n.1960), e o nipo-norte-americano Shuji Nakamura (n.1954) pela invenção dos Diodos-Emissores de Luz Azul ["Blue Light-Emittings Diodes" (LED)] que permitiram o brilho e a economia de energia das fontes de luz branca.

Palavras-chave: Prêmio Nobel de Física de 2014; Akasaki, Amano e Nakamura; LED azul.

\begin{abstract}
In this article, we will address the 2014 Nobel Prize in Physics awarded to the physicists: the japaneses Isamu Akasaki (n.1929), Hiroshi Amano (n.1960) and the japanese-american Shuji Nakamura (n.1954) for the invention of efficient Blue Light-Emittings Diodes (LED) which has enabled bright and energy-saving white light sources.
\end{abstract}

Keywords: 2014 Physics Nobel Prize; Akasaki, Amano e Nakamura; Blue LED.

\section{Introdução}

O Prêmio Nobel de Física de 2014 (PNF/2014) foi concedido aos físicos: os japoneses Isamu Akasaki (n.1929), Hiroshi Amano (n.1960) e Shuji Nakamura (n.1954) (naturaliza-

\footnotetext{
${ }^{+}$The 2014 Physics Nobel Prize

* Recebido: janeiro de 2015. Aceito: fevereiro de 2015.

${ }^{1}$ E-mail: jmfbassalo@gmail.com
} 
do norte-americano) pela invenção dos Diodos-Emissores de Luz Azul ["Blue LightEmittings Diodes" (LED)] que permitiram o brilho e a economia de energia das fontes de luz branca.

Inicialmente, tracemos uma pequena biografia dos nobelistas. Akasaki nasceu no dia 30 de janeiro de 1929, em Chiran, no Distrito Kawanabe, cidade da Prefeitura de Kagoshima, situada na ponta sudoeste da Ilha de Kyushu, no Japão. Ele se graduou na Universidade Kyoto, em 1952, e obteve o Doutorado em Engenharia Eletrônica, na Universidade Nagoya (UN), em 1964. Na década de 1960, ele trabalhou no Matsushita Research Institute Kokyo Incorporation (MRIK), época em que começou a trabalhar com o crescimento dos cristais de nitreto de gálio (GaN), fundamental para a invenção do LED azul. Em 2004, ele foi escolhido Professor Emérito da Nagoya University (NU) e, desde 2010, é Professor (Lifetime) da Graduate School of Science and Technology da Meijo University. Note-se que, com os royalties das patentes das invenções envolvendo o GaN e os LEDs decorrentes do trabalho de Akasaki (ver mais adiante), a NU inaugurou, em 06 de outubro de 2006, o Nagoya University Akasaki Institute (NUAI).

Antes do PNF/2014, Akasaki foi agraciado com as seguintes Honrarias [Distinção (Award), Medalha (Medal), Prêmio (Prize), Membro (Fellow), Doutor Honoris Causa etc.]: 1) Japanese Association for Crystal Growth (JACG) Award, 1989; 2) Chu-Nichi Culture Prize, 1991; 3) Technological Contribution Award (JACG), 1994; 4) Heinrich Welker Gold Medal (International Symposium on Compound Semiconductors), 1995; 5) Engineering Achievement Award [Institute of Electrical and Electronics Engineers (IEEE)/Lasers Electro-Optics Society], 1996; 6) Medal with Purple Ribbon [Japanese Government (JG)], 1997; 7) Inoue Harushige Award [Japan Science and Technology Agency (JSTA)], 1998; 8) C \& C Prize [Nippon Electric Company Corporation (NECC)], 1998; 9) Laudise Prize [International Corporation for Crystal Growth (ICCG)], 1998; 10) Jack A. Morton Award (IEEE), 1998; 11) Rank Prize [Rank Prize Foundation (RPF)], 1998; 12) Fellow (IEEE), 1999; 13) Gordon E. Moore Award [Electrochemical Society (ES)], 1999; 14) Honoris Causa Doctorage (Université Montpellier II), 1999; 15) Toray Science and Technology Prize [Toray Science Foundation (TSF)], 1999; 16) Asahi Prize [Asahi Shinbun Cultural Foundation (ASCF)], 2001; 17) Honoris Causa Doctorage (Linkoping University), 2001; 18) Outstanding Achievement Award [Japan Society of Applied Physics (JSAP)], 2002; 19) Fujihara Prize [Fujihara Foundation of Science (FFS)], 2002; 20) Takeda Award [Takeda Foundation (TF)], 2002; 21) Order of the Rising Sun, Gold Rays with Neck Ribbon (JG), 2002; 22) President's Award [Science Council of Japan (SCJ)]; 23) Solid State Devices \& Materials (SSDM) Award, 2003; 24) Tokai TV Culture Prize, 2004; 25) Person of Cultural Merit (JG), 2004; 26) John Bardeen Award [Minerals, Metals \& Materials Society (MMMS)], 2006; 27) Outstanding Achievement Award (JACG), 2006; 28) Honorable Lifetime Achievement Award [Japan Society for the Promotion of Science (JSPS)], 2007; 29) Foreign Associate [US National Academy of Engineering (US-NAE)], 2008; 30) Kyoto Prize Advanced Technology [Inamori 
Foundation (IF)], 2009; 31) Edison Medal (IEEE), 2011; 32) Special Award for Intellectual Property Activities (JSTA), 2011; 33) Minami-Nippon Culture Prize - Honorable Prize, 2011; e Order of Culture [Japanese Emperor (JE)], 2011.

O nobelista Amano nasceu no dia 11 de setembro de 1960, em Hamamatsu, cidade situada na parte oeste da Prefeitura de Shizuoka, no Japão. Ele estudou Física na UN, onde obteve o Bacharelado, em 1983, o Mestrado, em 1985, e o Doutorado, em 1989. Ainda como estudante de Física, em 1982, ele começou a participar do Grupo de Pesquisa do nobelista Akasaki, grupo esse que trabalhava com o crescimento do GaN, como vimos antes, e com o qual trabalhou entre 1988 e 1992, como Pesquisador Associado. Em 1992, ele foi a Universidade Meijo, primeiro como Professor Assistente e, de 1998 até 2002, como Professor Associado e, no período 2002-2010, como Professor Titular. A partir de 2010 (e até o momento), tornou-se Professor da Escola de Graduação de Engenharia, da UN.

O terceiro nobelista Nakamura nasceu no dia 22 de maio de 1954, em Ikata, do Distrito Nishiuwa, pequena cidade da Prefeitura Ehime, na Ilha de Shikoku, no Japão. Ele formou-se Bacharel em Engenharia Eletrônica, em 1977, na Universidade de Tokushima (UT), na mesma Universidade em que obteve o Mestrado em Engenharia Eletrônica, em 1979. Logo depois, foi trabalhar na Nichia Chemical Corporation [fundada pelo professor japonês Nobuo Ogawa (1912-2002), em 1956], em Tokushima, uma província localizada naquela Ilha japonesa. Nessa empresa, Nakamura começou a trabalhar com o GaN, buscando produzir um LED azul. Quando essa empresa começou a ter dificuldades econômicas, sua verba foi cortada, ele continuou sozinho em sua pesquisa, até 1993, quando finalmente o construiu, como veremos mais tarde. Em 1994, ele recebeu o título de Doutor em Engenharia, ainda pela UT. Em 1999, Nakamura deixou a Nichia e foi ocupar, em 2000, o cargo de professor do Departamento de Materiais da Universidade da California, em Santa Barbara (DM/UC/SB). Em 2005, naturalizou-se cidadão norte-americano e, em 2008, ele e os físicos norte-americanos Steven P. DenBaars e James S. Speck, também professores do DM/UC/SB fundaram a Soraa Incorporation, para desenvolver a tecnologia de LEDs.

Antes do PNF/2014, Nakamura foi agraciado com as seguintes Honrarias [Distinção (Award), Medalha (Medal), Prêmio (Prize), Honorary Doctor etc.]: 1) Asahi Prize (ASCF) (junto com Akasaki), 2001; 2) Benjamin Franklin Medal in Physics [Franklin Institute (FI)], 2002; 3) Finland's Millenium Technology Prize, 2006; 4) European Inventor Award [European Patent Office (EPO)], 2007; 5) Prince of Asturias Award for Technical and Scientific Research, 2008; 6) Honorary Degree of Doctor of Engineering (Hong Kong University of Science and Technology), 2008; 7) Harvey Prize (Technion, Israel), 2009; e 8) Inventor of the Year [Silicon Valley Intelectual Property Law Association (SVIPLA)], 2012.

Para entender a importância dos trabalhos desses nobelistas, façamos uma pequena revisão histórica sobre os semicondutores, base do primeiro LED ${ }^{1}$. Para isso, comecemos com

\footnotetext{
1 Charles Kittel, C. Introduction to Solid State Physics (John Wiley and Sons, 1971); John Michael Ziman, Principles of the Theory of Solids (Cambridge University Press, 1972); Rogério César de Cerqueira Leite;
} 
a Teoria de Bandas (parte da então Física do Estado Sólido, hoje denominada de Física da Matéria Condensada), fundamental para o estudo das propriedades condutoras, nãocondutoras e semicondutoras dos sólidos. O artigo pioneiro e fundamental da Teoria de Bandas foi escrito pelo físico suíço-norte-americano Felix Bloch (1905-1983; PNF, 1952)², em 1928. Em seu trabalho, Bloch assumiu que os elétrons se movimentam no condutor metálico sob a ação de um potencial periódico uni-dimensional, muito maior do que a energia cinética daqueles elétrons através do metal. Usando esse método - conhecido como ligações fortes ("tight-binding") -, Bloch resolveu a Equação de Schrödinger (ES) (1926) por intermédio da Análise de Fourier e da Teoria de Grupos, descobrindo com isso o hoje famoso Teorema de Bloch. Segundo esse Teorema, a função de onda tridimensional [ $\psi(\overrightarrow{\mathrm{r}})]$ [hoje conhecida como função (estado) de Bloch (FB)] de um elétron em um auto-estado de energia em uma rede periódica perfeita tem a forma do produto de uma onda plana [ $\exp (\mathrm{i} \overrightarrow{\mathrm{k}} \cdot \overrightarrow{\mathrm{r}})$ ] por uma função periódica $[u(\vec{r}+\vec{a})=u(\vec{r})]$, ou seja: $\psi(\vec{r})=u(\vec{r}) \times \exp (i \vec{k} \cdot \vec{r})$. Nessa expressão, $\vec{a}$ é o período da rede, $\vec{k}$ é o vetor de onda do cristal, e $\vec{r}$ é a coordenada do elétron. Note-se que, com essa função, Bloch mostrou existir uma relação entre a condutividade elétrica $(\sigma)$ e a temperatura absoluta $\mathrm{T}$, para baixas temperaturas.

Outro trabalho importante para o desenvolvimento da Teoria de Bandas foi o realizado pelo físico germano-norte-americano Hans Albrecht Bethe (1906-2005; PNF, 1967), em sua Tese de Doutorado, orientada pelo físico alemão Johannes Wilhelm Sommerfeld (18681951), na Universidade de Munique, na qual desenvolveu a Teoria do Campo Cristalino, e que foi publicada em 19283. Nessa Tese, Bethe estudou a difração de elétrons em cristais, usando uma aproximação de ligação fraca ("weak-binding"), para tratar a função (análoga a FB) de onda do elétron em um cristal de estrutura periódica. Ainda nessa sua Tese, Bethe demonstrou que para determinadas direções de incidência e para certos intervalos de energia do elétron, não se podiam construir soluções para sua propagação através do cristal. Isso significava que existiam regiões nas quais os elétrons não poderiam estar, isto é, as posteriormente conhecidas como Bandas Proibidas (BP).

Uma nova contribuição à Teoria de Bandas foi dada pelo físico inglês Rudolf Ernest Peierls (1907-1995) quando realizou seu trabalho de Doutoramento com o físico alemão Werner Karl Heisenberg (1901-1976; PNF, 1932), em Leipzig, a partir de 1928. Inicialmente, Heisenberg propôs como tema para a Tese de Peierls o estudo da condutividade, partindo da função de onda para um sistema de muitos elétrons, a fim de considerar a interação elétronelétron, uma vez que, no trabalho de Bloch acima referido, a função de onda do elétron único

Antônio Rubens Britto de Castro, Física do Estado Sólido (Editora Edgard Blüicher Ltda., 1978); Sílvio Roberto A. Salinas, Cadernos de História e Filosofia da Ciência, v. 3, p. 28, 1982; L. H. Hoddeson; G. Baym; M. Eckert, Reviews of Modern Physics, v. 59, p. 287, 1987; Tom Philbin, As 100 Maiores Invenções da História: Uma Classificação Cronológica (DIFEL, 2006).

${ }^{2}$ Zeitschrift für Physik, v. 52, p. 555, 1928.

${ }^{3}$ Annalen der Physik, v. 87, p. 55, 1928. 
por ele considerado, não permitia incorporar essa interação. Contudo, como Peierls não acreditasse que o modelo de multielétrons proposto por Heisenberg pudesse explicar a condutividade, Heisenberg sugeriu-lhe, então, o estudo das anomalias do Efeito Hall (EH). Registre-se que esse EH foi descoberto em outubro de 1879, pelo físico norte-americano Edwin Herbert Hall (1855-1938) realizou na Universidade Johns Hopkins, nos Estados Unidos da América do Norte, ao observar que quando uma longa lâmina de ouro ( $\mathrm{Au}$ ), percorrida longitudinalmente por uma corrente elétrica I, é colocada normalmente às linhas de força de um campo de indução magnética $\overrightarrow{\mathrm{B}}$ constante, surge, entre as laterais dessa mesma lâmina, uma diferença de potencial $\left(\mathrm{V}_{\mathrm{H}}\right)$, dada por: $\mathrm{V}_{\mathrm{H}}=\mathrm{I}_{\mathrm{H}}$, onde $\mathrm{R}_{\mathrm{H}}$ ficou conhecida como Resistência Hall. Muito embora essa voltagem concorde com alguns resultados experimentais envolvendo álcalis e certos metais [cobre $(\mathrm{Cu}), \mathrm{Au}$, prata $(\mathrm{Ag})$, chumbo $(\mathrm{Pb})$, paládio $(\mathrm{Pd})$ e manganês $(\mathrm{Mn})]$, no entanto, em outros metais [por exemplo, o bismuto (Bi)], há diferenças acentuadas em sua intensidade, assim como o sinal aparecia trocado. Além do mais, foi observado que $\mathrm{V}_{\mathrm{H}}$ variava com o módulo de $\overrightarrow{\mathrm{B}}$ e com a temperatura absoluta $\mathrm{T}$.

Assim, para estudar as anomalias do EH, Peierls considerou, em $1929^{4}$, que a variação temporal dos componentes do pacote de ondas de um elétron em campos elétrico e magnético é dada pela Força de Lorentz (1892). Nesses artigos, Peierls generalizou o trabalho de Bloch de 1928, ao assumir que a velocidade de grupo $\left(\mathrm{v}_{\mathrm{g}}\right)$ do elétron de energia (E), é dada por: $\mathrm{v}_{\mathrm{g}}=\mathrm{dE} / \mathrm{dk}$, onde $\mathrm{k}$ é o modulo do vetor de onda $(\overrightarrow{\mathrm{k}})$ do cristal (também conhecido como número de ondas), sendo $\hbar \overrightarrow{\mathrm{k}}=\overrightarrow{\mathrm{p}}$, o momentum linear do elétron. Peierls mostrou ainda que $\mathrm{v}_{\mathrm{g}}$ decrescia com $\mathrm{k}$, indicando completo desacordo com a hipótese do elétron livre; esse resultado significava dizer que o elétron se comportava como possuindo uma "massa efetiva" ( $\mathrm{m}$ *) $\left\{\mathbf{m}^{*}=(\hbar)^{2} /\left[\mathrm{d}^{2} \mathrm{E}(\mathrm{k}) / \mathrm{dk}^{2}\right]\right\}$, que pode ser negativa, em virtude da forma da função $\mathrm{E}(\mathrm{k})$. Desse modo, incluindo as colisões elétron-rede em sua generalização do trabalho de Bloch, Peierls explicou o EH.

Em virtude da viagem de Heisenberg a Chicago, nos Estados Unidos, na primavera de 1929, Peierls viajou então para Munique (recomendado por Heisenberg), para concluir seu Doutoramento com o físico austro-norte-americano Wolfgang Pauli Junior (1900-1958; PNF, 1945). Como já havia trabalhado em problemas de Física do Estado Sólido, Pauli sugeriu-lhe que estudasse a condução de calor em sólidos não-metálicos, isto é, isolantes. Desse modo, em outubro de 1929, Peierls conclui sua Tese de Doutoramento [publicada ainda em 19295], na qual fez uma análise crítica sobre o comportamento das vibrações da rede ("lattice") em equilíbrio térmico e em baixas temperaturas. Além do mais, ao introduzir o conceito de Processo Umklapp (do alemão: "girar sobre") (PU), encontrou que em um material puro, a conservação do momentum $(\overrightarrow{\mathrm{p}}=\hbar \overrightarrow{\mathrm{k}})$ do cristal que decorre da vibração da rede, implica que a condutividade térmica cresce exponencialmente com o decréscimo de $\mathrm{T}$.

\footnotetext{
4 Zeitschrift für Physik, v. 53, p. 255, 1929.

Physikalische Zeitschrift, v. 30, p. 273, 1929.

${ }^{5}$ Annales de Physique Leipzig, v. 3, p. 1055, 1929.
} 
Ainda como resultado de sua Tese de Doutoramento, em $1930^{6}$, Peierls usou as ideias que desenvolveu nessa Tese para estudar os metais. No entanto, diferentemente de Bloch (que havia considerado as vibrações da rede em equilíbrio térmico), Peierls escreveu as equações de Boltzmann (acopladas) tanto para elétrons quanto para as vibrações acústicas cristalinas, bem como considerou os dois casos limites - ligação forte ("tight") e ligação fraca ("weak") - para os elétrons nos sólidos. Além disso, admitiu, pela primeira vez, a aproximação de "íon rígido" para descrever a interação elétron-íon. Como resultado desse trabalho, Peierls demonstrou haver descontinuidades ("gaps") no espectro de energia eletrônico $\left[\mathrm{E}=(\hbar \mathrm{k})^{2} /(2 \mathrm{~m})\right]$ dos elétrons livres em um cristal sujeito a um potencial periódico fraco, em pontos para os quais se tem: $\mathrm{ka}= \pm \mathrm{n} \pi$, onde a é a periodicidade do cristal e $\mathrm{n}$ é um número inteiro.

A presença de descontinuidades (depois conhecidas como as BPs) no espectro de energia de elétrons sob potenciais periódicos foi também observada pelo físico norteamericano Philip McCord Morse (1903-1985). Vejamos de que maneira. No verão de 1929 , Morse trabalhava nos Bell Telephone Laboratories (BTL), nos Estados Unidos, sob a orientação de físico norte-americano Clinton Joseph Davisson (1881-1958; PNF, 1937), que lhe pediu que analisasse o resultado da experiência que ele fizera, em 1927, juntamente com o físico também norte-americano Lester Halbert Germer (1896-1971), sobre a difração de elétrons em superfícies metálicas. [Note-se que essa experiência comprovou a hipótese ondulatória que o físico francês, o Príncipe Louis Victor Pierre Raymond de Broglie (1894-1987; PNF, 1919), apresentou para o elétron, em 1924.] Em seu trabalho, Morse começou examinando as soluções gerais da ES para o elétron em um potencial periódico. Assim, usando equações análogas às empregadas por Bethe em sua Tese de Doutoramento, e considerou o potencial tridimensional a que o elétron está sujeito no interior de um metal, como uma soma de funções senoidais. Desse modo, em 19307, Morse demonstrou um importante resultado: A variação periódica do potencial dentro do cristal cria bandas proibidas (grifo meu) de energia mesmo para elétrons com energia maior que a máxima energia potencial.

Outro aspecto do comportamento de elétrons em metais foi obtido pelos físicos, o alemão Ralph de Laer Krönig (1904-1995) e o inglês Sir William George Penney (19091991), em $1931^{8}$, ao encontrarem a solução analítica para um modelo uni-dimensional de um potencial de poço quadrado. Nessa solução, eles obtiveram uma relação entre a estrutura de bandas e o espectro de energia dos estados quânticos de elétrons em cristais. Em 1931 e $1932^{9}$, o matemático e industrial inglês Sir Alan Herries Wilson (1906-1995) deu novas contribuições para o desenvolvimento da Teoria de Bandas, enquanto participava do grupo de

\footnotetext{
${ }^{6}$ Annales de Physique Leipzig, v. 4, p. 121, 1930.

${ }^{7}$ Physical Review, v. 35, p. 1310, 1930.

${ }^{8}$ Proceedings of the Royal Society of London A130, p. 499, 1931.

${ }^{9}$ Proceedings of the Royal Society of London A133; A134, p. 458; 277, 1931, 1932.
} 
Heisenberg, em Leipzig. Com efeito, ao estudar os trabalhos de Bloch e de Peierls, Wilson percebeu que o trabalho de Bloch (no qual os elétrons fortemente ligados poderiam mover-se através do metal), sugeria que todos os sólidos pudessem ser metais. Por outro lado, os trabalhos de Peierls sobre o EH indicavam, explicitamente, que uma banda cheia não conduzia corrente elétrica. Desse modo, e ainda baseado nos trabalhos de Bloch e Peierls, Wilson desenvolveu a ideia de que elétrons quase-livres, como os da Banda de Valência (BD) [estados esses que se formam em consequência da saída de elétrons dessa banda para a Banda de Condução (BC: região onde os elétrons livres circulam)] em átomos simples, poderiam formar camadas abertas ou fechadas. Além do mais, chegou ao curioso resultado de que assim como é possível obter condução com elétrons ligados, é possível, também, obter condução com elétrons livres.

Em consequência de suas pesquisas, Wilson fez a distinção clara entre condutores e não-condutores (isolantes), definindo-os, respectivamente, como sólidos que a banda de energia parcialmente cheia e, completamente cheia de elétrons, elétrons esses que obedecem ao Princípio da Exclusão de Pauli, de 1925 e à Estatística de Fermi-Dirac, de $1926^{10}$. Ainda para Wilson, os sólidos na Natureza, situados entre esses dois tipos - os chamados semicondutores - têm as bandas de energia ou quase cheias (ou quase vazias). É interessante ressaltar que a primeira evidência da existência de semicondutores ocorreu, em 1927, quando H. J. Seeman observou que o silício ( $\mathrm{Si}$ ) metálico, quando recoberto com uma camada de óxido, apresentava um aumento de condutividade. Logo depois, em 192811, o físico alemão Eduard Grüneisen (1877-1949) afirmou que, nesse tipo de sólido, a resistividade elétrica variava com o inverso de T. Por sim, em 1931, A. Schulze confirmou a observação de Seeman.

Muito embora Bloch tivesse (desde 1928, quando defendeu seu Doutoramento), outra opinião sobre a classificação dos sólidos com relação à condutividade elétrica, isto é, a de que a diferença entre condutores e isolantes era apenas quantitativa, pois dependia somente da facilidade com que um elétron poderia saltar de um átomo para outro, logo se convenceu das ideias de Wilson, que foram sumarizadas em um trabalho publicado, também, em $1931^{12}$. Neste trabalho, além de apresentar a diferença entre condutor e isolante, Bloch demonstrou que a presença de impurezas em um semicondutor faz aparecerem níveis de energia na BP. É importante observar que foi Heisenberg que, ainda em $1931^{13}$, tratou esses estados desocupados como buracos ("holes") e com existência própria, isto é, uma entidade física carregada positivamente. Com efeito, ao descrever um buraco ("lacuna") por intermédio de uma função de onda complexa, Heisenberg demonstrou que as "vacâncias" ("lacunas") próximas ao topo da BV, se comportavam exatamente como se fossem elétrons carregados positivamente, sob a

\footnotetext{
10 BASSALO; J. M.; CARUSO, F. Dirac/Fermi/Pauli. São Paulo: Livraria da Física, 2013.

${ }^{11}$ Handbuch der Physik, v. 13, p. 1, 1928.

12 Physikalische Zeitschrift, v. 32, p. 881, 1931.

13 Annales de Physique Leipzig, v. 10, p. 888, 1931.
} 
ação de um campo elétrico externo. Note-se que o conceito de buraco ("lacuna" ou "vacância") utilizado por Heisenberg não era novo em Física, já que o físico inglês Paul Adrien Maurice Dirac (1902-1984; PNF, 1933) o havia formulado, em 193014, em seu famoso "mar" de elétrons com energia negativa seus trabalhos sobre a Teoria Quântica do Elétron ${ }^{15}$.

Em seus trabalhos sobre a condutividade elétrica nos sólidos, realizados em 1931 e vistos acima, Wilson estudou as propriedades condutiva e óptica do óxido cúprico $(\mathrm{CuO})$. Nesse estudo, ele observou que, enquanto esse óxido apresentava uma banda de energia para absorção óptica como sendo em torno de 2 Volts, a sua energia de excitação elétrica era de apenas 0,6 Volts. Em vista disso, concluiu que essa "condutividade elétrica era devida à presença de impurezas". Assim, para Wilson, tal condutividade era devida ao elétron associado à impureza, cuja energia situava-se na BP próxima da $\mathrm{BC}$, de modo que tal elétron poderia ser excitado termicamente até esta BC. Assim, para Wilson, a condutividade de semicondutores pela presença de impurezas intrínsecas (defeitos) [ou mesmo pela ausência (vacância) de átomos do próprio cristal] em sua rede cristalina também foi observada em experiências realizadas por Peierls, em $1932^{16}$ e pelo físico suíço-alemão Walther Hermann Schottky (18361956), em $1933^{17}$ (com a colaboração de F. Waibel). É oportuno ressaltar que, em 192318, Schottky estudou a extração de elétrons em metais frios por intermédio de campos elétricos intensos, ocasião em que descobriu que a emissão termiônica (fluxo de elétrons) era aumentada quando um campo elétrico (E) atuava na superfície emissora. Desse modo, encontrou a dependência da corrente eletrônica (I) com E, isto é: $\mathrm{I} \propto \mathrm{E}^{1 / 2}$, relação essa que ficou mais tarde conhecida como Efeito Schottky (ESch), e que já havia sido previsto pelo físico austrohúngaro-norte-americano Julius Edgar Lilienfeld (1881-1963), em 192219.

Os tipos de experiências que analisamos acima sobre semicondutores, nos três primeiros anos da década de 1930, foram sendo ampliadas e modificadas por toda essa década e na década de 1940, principalmente com o silício (Si) e o germânio (Ge). Com efeito, ao ser desenvolvida a técnica de dopagem, ou seja, a dissolução de traços de materiais quimicamente diferentes nesses dois tipos de semicondutores foi possível torná-los condutores. Porém, dependendo da "impureza" utilizada, tais semicondutores comportavam-se diferentemente com relação à condução. Por exemplo, o Ge e o Si são elementos químicos de valência 4 (número de elétrons em uma camada eletrônica bohriana, sendo que um deles pode ser compartilhado entre átomos). Por sua vez, o fósforo (P) e o arsênio (As), têm valência 5. Assim, se o Ge (ou $\mathrm{Si}$ ) for dopado com impureza do tipo P (ou As), o elétron extra que sobra (no

\footnotetext{
14 Proceedings of the Royal Society of London A133, p. 360, 1930.

15 BASSALO; J. M.; CARUSO, F. Dirac. São Paulo: Livraria da Física, 2013.

BASSALO; J. M.; CARUSO, F. Heisenberg. São Paulo: Livraria da Física, 2014.

16 Ergebnisse der Exacten Naturwissenschaften, v. 11, p. 264, 1932.

${ }^{17}$ Physikalische Zeitschrift, v. 34, p. 858, 1933.

18 Zeitschrift für Physik, v. 14, p. 63, 1923.

${ }^{19}$ Physikalische Zeitschrift, v. 23, p. 306, 1922.
} 
caso, o quinto) será responsável pelas propriedades condutoras do Ge (ou do $\mathrm{Si}$ ) que, neste caso, recebe o nome de semicondutor tipo-n, onde n significa que o "portador de carga" é negativo. Por sua vez, se o $\mathrm{Ge}$ (ou Si) for dopado com uma impureza cuja valência é menor do que 4, a ausência do elétron do átomo inserido no cristal semicondutor cria um sítio vazio [lacuna ou buraco ("hole")] para o qual se dirige um elétron vizinho daquele cristal. Por outro lado, para esse novo buraco, se dirige um novo elétron e, assim sucessivamente. Portanto, tudo se passa como se o buraco caminhasse no semicondutor, semelhante a uma carga positiva. Por exemplo, isso acontece se o Ge (ou Si) for dopado com átomos de valência 3 , tais como o gálio (Ga). Neste caso, o semicondutor recebe o nome de semicondutor tipo-p, onde p significa que o "portador de carga" é positivo. É interessante registrar que, em 1938, baseado em seu efeito, Schottky desenvolveu uma teoria para explicar o comportamento retificante do contato entre um metal [p.e.: alumínio ( $\mathrm{A} \ell$ )] e um tipo-n (Si). Como esse tipo de contato tem o mesmo papel do então tubo termiônico \{depois: válvula termiônica ou diodo [conjunto de dois eletrodos (catodo e anodo) no vácuo\}, inventada pelo físico e engenheiro eletricista inglês Sir John Ambrose Fleming (1849-1945), em 1904, esse contato passou a ser conhecido como diodo Schottky (DS).

A importância tecnológica dos semicondutores dopados surgiu nas célebres experiências realizadas nos Bell Telephone Laboratories (BTL) pelos físicos norte-americanos John Bardeen (1908-1991; PNF, 1956; 1972), William Bradford Shockley (1910-1989; PNF, 1956) (de origem inglesa) e Walter Houser Brattain (1902-1987; PNF, 1956), na segunda metade da década de 1940. Com efeito, em 1945 [Electrons and Holes in Semiconductors (D. Van Nostrand Company, Incorporation, 1950)], Shockley descobriu que um cristal de Ge contendo traços de uma impureza funcionava como retificador. Desse modo, poderia controlar os elétrons móveis no interior desse tipo de semicondutor, com um campo elétrico externo. Por sua vez, em 1947 (Physical Review 71, p. 717), Bardeen explicou essa descoberta de Shockley afirmando que a mesma era devido ao fato de existirem estados (gaps: "armadilhas") na superfície do Ge e, portanto, uma grande fração da carga induzida era imobilizada por tais "estados superficiais", estados esses que explicavam os aspectos "embaraçosos" (nenhuma diferença de potencial) no ponto de contato (diodo) entre os tipos n-p do Ge ou do Si. Ainda em 1947, Bardeen e Brattain imergiram uma peça de Ge em um eletrólito e descobriram que poderiam fazer passar uma corrente elétrica através de um material de alta resistência, fenômeno esse que passou a ser conhecido como Efeito Transistor (ET) ("Transfer Resistor"). Em 27 de dezembro de $1947^{20}$, Bardeen e Brattain usaram o ET para construir o transistor de pontas ("bigode de gato") constituído de uma base de Ge (tipo-n), na qual se apoiavam dois finos contactos metálicos. Um dos contactos era polarizado para frente em relação à base, compondo o denominado emissor. O segundo contacto apresentava uma polarização reversa, constituindo-se no coletor. Esse sistema funcionava como um amplificador. Em janeiro de 1948, Shockley inventou o transistor de junção, constituído por um "sandwich" de semiconduto-

${ }^{20}$ Physical Review 74, p. 230, 1948. 
res: n-p-n ou p-n-p (denominado mais tarde de triodo). Note-se que esse dispositivo funcionava como amplificador e retificador (Shockley, op. cit.).

Visto o pequeno histórico sobre os diodos e os triodos, retornemos aos trabalhos que levaram ao PNF/2014. Para isso, usaremos os textos da referência ${ }^{21}$; os sites da referência $^{22}$; além dos perfis dos nobelistas ${ }^{23}$.

O primeiro LED foi anunciado, em $1923^{24}$ [e confirmado em 192725], pelo físico russo Oleg Vladimirovich Losev (Lossev/Lossew) (1903-1942) ao observar que quando uma corrente contínua, oriunda de uma bateria, passava em cristais de carbeto de silício ( $\mathrm{SiC}$ ) (carborundum), um flash de luz verde aparecia. Muito embora esse fenômeno (eletroluminescência) já tivesse sido observado, em 190726, pelo inventor inglês, o Capitão Henry Joseph Round (1881-1966) [assistente pessoal do físico italiano Guglielmo Marconi (1874-1937; PNF, 1909)], foi Los(s)ev(w) quem o entendeu, propôs uma teoria para explicá-lo e o usou em dispositivos eletroluminescentes, segundo ele registrou em seus artigos ${ }^{27}$.

Muito mais tarde, já na década de 1950, novas evidências da eletroluminescência foram anunciadas. Com efeito, em 195128, os engenheiros-físicos norte-americanos Kurt Lehovec (1918-2012) (nascido na Bohemia), Carl Anthony Accardo (1928-2014) e Edward Jamgochian (n.1924) que trabalhavam no Signal Corps Engineering Laboratory (SCEL), explicaram a eletroluminescência observada por Lehovec como resultado da injeção de portadores de carga (carriers) através de uma junção p-n (que ocorre no SiC) seguida de uma radiação luminosa devido a recombinação de elétrons e buracos. Porém, como a energia do fóton observado era menor do que a energia da junção (bandgap $=1,4 \mathrm{eV}$ ), eles sugeriram que aquela radiação ocorria por causa de impurezas ou defeitos do cristal de SiC. No ano seguinte, em 195229, os físicos norte-americanos J. R. Haynes e W. C. Westphal, trabalhando no BTL, analisaram a mobilidade de elétrons no $\mathrm{Si}$. Logo depois, em $1955^{30}$, o físico norte-americano Rubin Braunstein, que trabalhava na Radio Corporation of America (RCA) (em Princeton, New Jersey, nos Estados Unidos) observou uma emissão infravermelha em heterojunções

21 VIEIRA, C. L. (Entrevistador). Shuji Nakamura: O Novo Edison. Ciência Hoje, v. 39, p. 6, ago. 2006; GARCIA, M. Luzes do Novo Século [CH (On-line), 08 out. 2014]; e Class for Physics of the Royal Swedish Academy of Sciences, 07 out. 2014.

22 <www.sslighting.net/maruska_blue_led_history.pdf; wikipedia.org/light_emitting_diode>. Neste site o leitor poderá encontrar uma série de aplicações atuais de todos os tipos de LEDs, inclusive o LED branco.

23 <wikipedia.org/Isamu_Akasaki/Hiroshi-Amano/Shuji_Nakamura>

${ }^{24}$ Telegrafiya i Telefoniya bez Provodov, v. 18, p. 61, 1923.

25 Telegrafiya i Telefoniya bez Provodov, v. 44, p. 485, 1927.

${ }^{26}$ Electrical World, v. 19, p. 309, 1907.

${ }^{27}$ Philosophical Magazine, v. 6, p. 1024, 1928; USSR Patent 12191 (1929); Comptes Rendus de l’Académie de Sciences de Paris, v. 39, p. 363, 1940.

${ }^{28}$ Physical Review, v. 83, p. 603, 1950.

${ }^{29}$ Physical Review, v. 85, p. 680, 1952.

${ }^{30}$ Physical Review, v. 99, p. 1892, 1955. 
semicondutoras (HeS) (envolvendo elementos químicos das colunas III-V da Tabela Periódica dos Elementos) tais como: gálio-arsênio (GaAs: arseneto de gálio); gálio-antimônio (GaSb); gálio-nitrogênio (GaN: nitreto de gálio); índio-fósforo (InP: fosfeto de índio) e silício-germânio (SiGe). Resultados análogos foram publicados por G. A. Wolff, R. A. Herbert e J. D. Broder, também em 1955² . Ainda em 1955, Haynes ${ }^{32}$ e, em 1956, Haynes e Westphal ${ }^{33}$, mostraram que a eletroluminescência percebida na junção p-n ( $\mathrm{SiGe}$ ) também se devia à recombinação de elétrons e buracos. Resultados semelhantes foram obtidos, em $1958^{34}$, por D. A. Holt, G. F. Alfrey e C. S. Wigglins.

Registre-se que as HeS foram desenvolvidas, independentemente, pelos grupos dos físicos liderados pelo alemão Herbert Kroemer (n.1928; PNF, 2000) e o russo Zhores I. Alferov (n.1930; PNF, 2000). Kroemer desenvolveu seu trabalho em vários lugares: em 1953, no Fernmeldetechnisches Zentralamt - FTZ ("Laboratório Central de Telecomunicações”) do Serviço Postal Alemão; em 1954, na RCA; entre 1963-1966, na Varian Associates, no Silicon Valley, em Palo Alto, Califórnia; e, em 1968, foi para a Universidade de Colorado, onde permaneceu até 1976, quando então se deslocou para a Universidade da Califórnia, em Santa Bárbara, para ser professor no Departamento de Engenharia Elétrica e Engenharia Computacional. Por sua vez, Alferov desenvolveu suas pesquisas nas $\mathrm{HeS}$, no hoje famoso Instituto Físico-Técnico A. F. Ioffe (IFT-AFI), em Leningrado, que havia sido organizado pelo físico russo Abram Fedorovic Ioffe (1880-1960), em 1919, como a Faculdade de Física e Mecânica do Instituto Politécnico de São Petersburgo. Para mais detalhes sobre as HeS, ver: Herbert Kroemer, Autobiography e Nobel Lecture: Quasi-Electric Fields and Band Offsets: Teaching Electrons new Tricks (Nobel e-Museum, 08 de dezembro de 2000), e Zhores I. Alferov, Autobiography e Nobel Lecture: The Double Heterostructure: Concept and its Applications in Physics, Electronics and Technology (Nobel e-Museum, 08 de dezembro de 2000).

Agora, vejamos como aconteceu a invenção dos LEDs, objeto deste artigo. Essa invenção dependeu de várias técnicas de crescimento de cristais envolvendo as HeS, e que foram desenvolvidas em vários laboratórios do mundo, com diferentes objetivos: telefonia, iluminação e televisão. O primeiro cristal considerado foi o $\mathrm{GaN}$ [bandgap: 3,4 eV; emissão de luz ultravioleta $\left(\lambda<400 \mathrm{~nm} ; 1 \mathrm{~nm}=10^{-9} \mathrm{~m}\right)$ ], por pesquisadores da Phillips Central Laboratory (PLC), na Alemanha $\left\{\right.$ H. G. Grimmeiss e H. Koelmans ${ }^{35}$ [DPB (patente alemã) 1.077.330 (1960) (com I. B. Maak)]\}. Esse tipo de cristal pode ser crescido em substrato de safira $\left(\mathrm{A}_{2} \mathrm{O}_{3}\right)$ ou $\mathrm{SiC}$ e pode ser dopado com $\mathrm{Si}$ (tipo-n) ou magnésio $(\mathrm{Mg}$ ) (tipo-p), porém esses dopadores tornam aquele cristal muito frágil.

\footnotetext{
${ }^{31}$ Physical Review, v. 100, p. 1144, 1955.

32 Physical Review, v. 98, p. 1866, 1955.

33 Physical Review, v. 101, p. 1676, 1956.

34 Nature, v. 181, p. 109, 1958.

35 Zeitschrift für Naturforschung 14a, p. 264, 1959; 15, p. 799, 1960.
} 
No outono de 1961, os inventores (engenheiros elétricos) norte-americanos James R. ("Bob") Biard (n.1931) e Gary E. Pittman, enquanto trabalhavam no Texas Instruments Incorporation (TII), em Dallas, registraram que o GaAs emitia radiação infravermelha quando uma corrente elétrica era aplicada. Em 08 de agosto de 1962, eles conseguiram uma Patente (US3293513) pela invenção do Semiconductor Radiant Diode (SRD). Note-se que, em outubro de 1962, o TII anunciou o primeiro LED comercial (SNX-100), que empregava um cristal puro de GaAs que emitia radiação $\operatorname{com} \lambda=900 \mathrm{~nm}$.

Novas técnicas mais eficientes de junções p-n $\left(\mathrm{GaP}_{\text {e }} \mathrm{GaP}_{\mathrm{x}} \mathrm{As}_{1-\mathrm{x}}\right)$ \{ usando diferentes dopantes [p.e.: zinco (Zn) e N]\} foram rapidamente desenvolvidas ainda na década de 1960, com diferentes objetivos: telefonia, iluminação e televisão, segundo já registramos. Destaquem-se os trabalhos desenvolvidos pelos seguintes grupos de pesquisa: 1) PCL \{Grimmeiss e Koelmans ${ }^{36}$; Grimmeiss e H. Scholz ${ }^{37}$ \}; 2) Services Electronics Laboratories, na Inglaterra \{J. Starkiewicz e J. W. Allen ${ }^{38}$ \}; e 3) BTL \{M. Gershenzon e R. M. Mikulyak ${ }^{39}$ \}. Note-se que no verão de 1962, o físico russo-norte-americano Jacques I. Pankove ${ }^{40}$ (n.1922), reportou que havia observado emissão de luz infravermelha em junções p-n.

Apesar dessas observações sobre a emissão de radiação por parte de junções p-n (diodos), a invenção do primeiro LED (no espectro vermelho visível da luz) aconteceu no laboratório da empresa General Electric Corporation (GEC), em Syracuse (New York) e se deve ao físico norte-americano Nick Holonyak Junior (n.1928). Com efeito, em 196241, ele e Sam F. Bevacqua anunciaram que, usando junções de semicondutores de Ga e As, com uma mistura de $\mathrm{P}\left(\mathrm{GaAs}_{1-\mathrm{x}} \mathrm{P}_{\mathrm{x}}\right)$, observaram um flash vermelho. (Aliás, é interessante registrar que Holonyak, no volume de fevereiro de 1963 do Reader'd Digest, previu que os LEDs iriam substituir as lâmpadas incandescentes edisonianas, conforme está sendo gradualmente realizado no mundo atual.) É oportuno destacar que, ainda em 1962, Robert N. Hall, G. E. Fenner, J. D. Kingsley, T. J. Soltys e R. O. Carlson ${ }^{42}$ da GEC, em Schenectady; Marshall I. Nathan, W. P. Dumke, G. Burns, F. H. Dill Junior e G. Lasher ${ }^{43}$ da International Business Machines Corporation (IBM), em Watson; R. J. Keyes e T. M. Quist ${ }^{44}$ e Quist, Robert H. Redi-

\footnotetext{
36 Physical Review, v. 123, p. 1939, 1961.

37 Physics Letters, v. 8, p. 233, 1964.

38 Journal of Physics and Chemistry of Solids, v. 23, p. 881, 1962.

39 Journal of Applied Physics, v. 32, p. 1338, 1961.

40 Physical Review Letters, v. 9, p. 283; Proceedings of the Institute of Radio Engineers, v. 50, p. 1976 (com a colaboração de J. E. Berkeyheiser).

41 Applied Physics Letters, v. 1, p. 82, 1962.

42 Physical Review Letters, v. 9, p. 366, 1962.

43 Applied Physics Letters, v. 1, p. 62, 1962.

44 Proceedings of the Institute of Radio Engineers, v. 50, p. 1822, 1962.
} 
ker, R. J. Keyes, W. E. Krag, B. Lax, A. L. McWhorter e H. J. Zeiger5, do Lincoln Laboratory, do Massachusetts Institute of Technology (LL/MIT), também registraram o mesmo flash vermelho, porém usando apenas o composto semicondutor GaAs.

Ainda na década de 1960, verificou-se que o GaP (fosfeto de gálio) não era tão eficiente como emissor de luz quanto o GaAs. Este, por sua vez, se tornava muito menos eficiente $(0,005 \%)$ quando a concentração de $\mathrm{P}$ excedia a $44 \%$ conforme o físico norte-americano Herbert Paul Maruska (n.1944) e Pankove mostraram em 196746. Logo depois, em 196847, R. A. Logan, H. G. White e W. Wiegmann anunciaram que haviam obtido um LED amareloverde $(550 \mathrm{~nm})$ usando uma junção GaP dopada com $\mathrm{N}$ (GaP:N).

No final da década de 1960, a RCA fabricava as primeiras televisões (TV) coloridas usando tubos de raios catódicos tradicionais. E, para isso, contava com a colaboração de uma de suas Divisões, a Materials Research Division (MRD), então dirigida pelo físico-químico norte-americano James T. Tietjen (n.1933) e que desejava construir uma televisão colorida plana usando LEDs. Ora, como a televisão a cores é baseada nas três cores básicas (com $\lambda$ dado em $\mathrm{nm})$ : vermelho $(610<\lambda<760)$, verde $(500<\lambda<570)$ e azul $(450<\lambda<500)$, e considerando que o LED vermelho já tinha sido construído em 1962, usando $\mathrm{GaP}_{\mathrm{x}} \mathrm{As}_{1-\mathrm{x}}$ e que o LED amarelo-verde acabara de ser construído, em 1968, com GaP:N, como vimos acima, faltava apenas construir o LED azul. Assim, em 03 de maio de 1968, Tietjen conversou com Maruska, membro de sua equipe na MRD qque construía filmes cristalinos de GaP:N para obter o LED vermelho usando a técnica HVPE [Hydride (Halide) Vapor Phase Epitaxy] \} e sugeriu-lhe que usasse essa mesma técnica para crescer filmes cristalinos de GaN, pois esperava, com tais cristais, obter o LED azul. Motivado por esse desafio, Maruska foi a busca de informações sobre o GaN, na Biblioteca David Sarnoff Research Center, da RCA, e xerocou os trabalhos dos químicos alemães Robert Juza e Harry Hahn, de $1938^{48}$, que tratava do GaN. [É interessante destacar que, como a RCA estava fazendo economia, ele usou a face não usada de xerox (que datavam de 03/05/1968) para tirar tais cópias e, por isso, essa data marca o início do programa da RCA do desenvolvimento da técnica de crescimento de filmes de GaN, por intermédio da HVPE.] Contudo, para esse crescimento, havia um pequeno problema, pois até então, todos os filmes de $\mathrm{GaN}$ eram crescidos em temperaturas abaixo de $600{ }^{\circ} \mathrm{C}$, muito inferior a temperatura típica de $850{ }^{0} \mathrm{C}$ de crescimento do GaAs. Para contornar esse problema, em 05 de março de 1969, Maruska substituiu o vácuo por um banho de amônia $\left(\mathrm{NH}_{3}\right)$ e conseguiu, na temperatura de $950{ }^{\circ} \mathrm{C}$, obter um filme fino de $\mathrm{GaN}$ e o resultado desse trabalho de pesquisa foi apresentado por ele e Tietjen, em 196949. Como os filmes de GaN assim obti-

\footnotetext{
45 Applied Physics Letters, v. 1, p. 91, 1962.

46 Solid State Electronics, v. 10, p. 917, 1960.

47 Applied Physics Letters, v. 13, p. 139, 1968.

48 Zeitschrift für Anorganische und Allgemeine Chemie, v. 239, p. 282, 1938.

${ }^{49}$ Applied Physics Letters, v. 15, p. 327, 1969.
} 
dos eram naturalmente (sem dopantes) do tipo-n, alguns laboratórios do mundo (Europa, Japão e inclusive a RCA) procuravam encontrar dopantes que fossem do tipo-p para, então, realizar uma junção p-n. Como o Zn já havia sido usado em outras HeS, houve uma tentativa de frustrada de aproveitá-lo para obter tal junção.

Depois de passar seu ano sabático de 1969 na Berkeley University, Pankove voltou para a RCA, em janeiro de 1970 e, sabendo do trabalho de Maruska sobre o crescimento do $\mathrm{GaN}$, imediatamente se juntou a ele e formaram um grupo de pesquisa para trabalhar na absorção óptica e fotoluminescência de filmes finos de GaN. Essa união resultou nos artigos que foram apresentados, ainda em $1970^{50}$.

Já com 26 anos de idade, Maruska foi realizar seu Doutorado na Stanford University (SU), em 1970, com uma bolsa de estudos da RCA, com a finalidade de trabalhar na busca do LED azul. Enquanto isso, na RCA, Pankove continuou suas pesquisas com o crescimento eletroluminescente de filmes de GaN dopados com Zn e In. Assim, em 1971, a RCA apresentou o LED (GaN-Zn) com um pico de azul-brilhante $(475 \mathrm{~nm})^{51}$ e o LED (GaN-In) ${ }^{52}$. Registre-se que, ainda em 197153, R. Dingle, D. D. Sell, S. E. Stokowski e M. Ilegems usaram a técnica do HVPE para estudar a absorção, a refletância e a luminescência do GaN.

Depois de realizar seu Exame de Qualificação, Maruska e seu colega de doutorado, o engenheiro norte-americano Walden C. ("Wally") Rhines (n.1946) foram trabalhar com o físico norte-americano David A. Stevenson (1928-1994), que dirigia o Materials Science Department, da SU e, juntos, construíram um reator do tipo RCA-HVPE. Em abril de 1972, Maruska teve a ideia de usar o $\mathrm{Mg}$ como um dopante do tipo-p nos filmes de GaN e, em 07 de abril de $1972^{54}$, ele, Rhines e Stevenson anunciaram que haviam construído um LED (GaN$\mathrm{Mg}$ ) violeta-brilhante $(430 \mathrm{~nm})$, cuja patente americana foi-lhes concedida em 1974 (US3819974 A). Observe-se que, um ex-aluno de Holonyak, o engenheiro elétrico norteamericano M. George Craford, trabalhando na Monsanto Chemical Company, inventou, ainda em 1972, o primeiro LED amarelo, e melhorou, em dez (10) vezes, o brilho dos LED vermelho e vermelho-laranja. Observe-se também que Maruska, Stevenson e Pankove detalharam o LED (GaN-Mg), em 197355. Note-se ainda que, apesar do relativo sucesso da técnica HVPE, esta apresentava algumas dificuldades na formação de doadores tipo-p, pois não se entendia bem o papel do hidrogênio $(\mathrm{H})$, pois ele se apresentava inerte naquela formação, con-

\footnotetext{
50 Solid State Communications, v. 8, p. 1051, 1970 (Pankove, Berkeyheiser, Maruska e J. Wittke); Applied Physics Letters, v. 7, p. 197, 1970 (Pankove, Maruska e Berkeyheiser).

51 Journal of Luminescence, v. 4, p. 63, 1971 (Pankove, E. A. Miller, D. Richman e Berkeyheiser).

52 RCA Review, v. 32, p. 383, 1971 (Pankove, Miller e Berkeyheiser).

53 Physical Review B4, p. 1211, 1971.

54 Material Research Society Bulletin, v. 7, p. 777, 1972.

55 Applied Physics Letters, v. 22, p. 303, 1973.
} 
forme Pankove destacou, em 197356. Ainda nesse ano de 1973, alguns trabalhos foram publicados envolvendo o uso daquela técnica ${ }^{57}$.

Em 1974, a equipe RCA-SU desenvolveu uma nova técnica envolvendo o tunelamento de elétrons [emitidos por metais frios sob a ação de campos elétricos fortes, conforme foi demonstrado pelos físicos, o inglês Sir Ralph Howard Fowler (1899-1944) e o alemão Lothar Wolfgang Nordheim (1899-1985), em 192858, conhecido como Efeito FowlerNordheim (EF-N)] através de uma barreira triangular de potencial e que apresentava a característica de serem independentes da temperatura. Desse modo, seus membros propuseram, ainda em $1974^{59}$ encontrar uma luminescência (LED) azul devido ao impacto de elétrons quentes em junções dopadas com Mg. Contudo, com a crise econômica da RCA ocorrida no começo de 1974, seu projeto de construir o LED azul foi cancelado, mas a técnica HVPE para crescimento de filmes de GaN ainda foi usada na década de 1970, como se pode ver nos seguintes artigos: em 197060, por Grimmeiss e B. Monemar; em 197461, por Monemar; e, em $19766^{62}$, por Masahiko Sano e M. Aoki. Observe-se que, ainda em 197663, T. P. Pearsall, B. I. Miller, R. J. Capik e K. J. Bachmann criaram o primeiro LED [infravermelho $(1.100 \mathrm{~nm})$ ] de brilho alto-eficiente usando uma liga de materiais semicondutores $\left(\mathrm{InP} / \mathrm{Ga}_{0.17} \mathrm{In}_{0.83} \mathrm{As}_{0.34} \mathrm{P}_{0.66} / \mathrm{InP}\right)$ especificamente adaptados para ser usado em telecomunicações por fibra óptica. È ainda oportuno destacar que, também na década de 1970, duas novas técnicas para crescimento de cristais foram inventadas: a MOVPE ("Metalorganic Vapour Phase Epitaxy”), em 197164, por H. M. Manasevit, F. M. Erdmann e W. I. Simpson; e a MBE ("Molecular Beam Epitaxy"), em 197565, por A. Y. Cho e J. R. Arthur. Note-se que, em 198366, S. Yoshida, S. Misawa e S. Gonda usaram essas técnicas para crescimento do GaN.

Completemos este artigo, analisando os trabalhos dos nobelistas que levaram à invenção do LED azul brilhante. Na década de 1960, Akasaki começou a trabalhar no MRIK, com o crescimento dos cristais (junção p-n), o cristal de GaN, usando a HVPE. Em 1981,

\footnotetext{
56 Journal of Luminescence, v. 7, p. 114, 1973.

57 Journal of Luminescence, v. 8, p. 89, 1973 (Pankove, Miller, Richman e Berkeyheiser); Applied Physics Letters, v. 22, p. 303, 1973 (Maruska, Stevenson e Pankove).

58 Proceedings of the Royal Society of London A119, p. 173, 1928.

59 Journal of Electrochemical Society, v. 121, p. 1202, 1974 (Maruska, Anderson e Stevenson); Solid State Electronics, v. 17, p. 1171, 1974 (Maruska e Stevenson); Physical Review Letters, v. 33, p. 361, 1974 (Pankove e M. A. Lampert).

${ }^{60}$ Journal of Applied Physics, v. 41, p. 4054, 1970.

61 Physical Review B10, p. 676, 1974.

62 Japanese Journal of Applied Physics, v. 15, p. 1943, 1976.

63 Applied Physics Letters, v. 28, p. 499, 1976.

64 Journal of The Electrochemical Society, v. 118, p. 1864, 1971.

65 Progress in Solid State Chemical, v. 10, p. 157, 1975.

66 Applied Physics Letters, v. 42, p. 427, 1983.
} 
agora na Nagoya University (NU), Akasaki continuou a trabalhar com aquela técnica, cujo resultado foi apresentado por ele, Y. Ohki, Y. Toyoda e H. Kobayasi, no Ninth International Symposium on Gallium Arsenide and Related Compunds, realizado em Oiso, no Japão, em 198167. Logo em 1982, Amano (recém formado) juntou-se ao seu grupo e, nas décadas de 1980 e 1990, obtiveram resultados importantes para a obtenção do LED azul brilhante, tais como: a) $1986^{68}$; b) $1988^{69}$; c) $1989^{70}$; d) $1990^{71}$; e) $1991^{72}$; f) $1992^{73}$; g) $1995^{74}$; h) $1996^{75}$; i) $1997^{76}$; j) $1999^{77}$; k) $2000^{78}$; 1) $2000^{79}$; m) $2001^{80}$; n) $2002^{81}$; e o) $2014^{82}$.

Para detalhes dos trabalhos de Akasaki e Amano, ver suas Nobel Lectures: Isamu Akasaki, Fascinated Journeys into Blue Light (08/12/2014, Stockholm University) e Hiroshi Amano, Growth of GaN on Sapphire by Low Temperature Deposited Buffer Layer

\footnotetext{
67 Proceedings, v. 63, p. 479, 1981.

68 Applied Physics Letters, v. 48, p. 353, 1986 (Amano, Nobuhiko Sawaki, Akasaki e Y. Toyoda).

69 Journal of Luminescence, v. 40\&41, p. 121, 1988 (Amano, Akasaki, T. Kozawa, Kasumasa Hiramatsu, Sawaki, K. Ikeda e Y. Ishii).
}

70 Japanese Journal of Applied Physics, v. 28, p. L2112, 1989 [Amano, M. Kito, Hiramatsu e Akasaki: neste trabalho, a dopagem do GaN com Mg foi realizada com a técnica LEEBI ("Low-Energy Electron Beam Irradiation”)]; Journal of Crystal Growth, v. 98, p. 209, 1989 (Akasaki, Amano, Yasuo Koide, Hiramatsu e Sawaki).

71 Materials Research Society: Extended Abstract, p. 165, 1990 (Amaro e Akasaki); Japanese Journal of Applied Physics, v. 29, p. L205, 1990 (Amano, Tsunemori Asahi e Akasaki).

72 Journal of Crystal Growth, v. 115, p. 648, 1991 [Hiramatsu, S. Itoh, Amano, Akasaki, N. Kuwano, T. Shiraishi e K. Oki: neste trabalho o crescimento do GaN ocorreu em safira usando uma fina camada (nm) do poli-

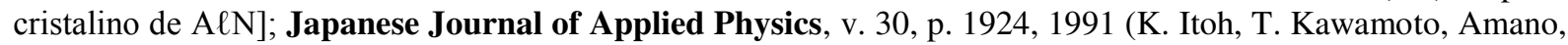
Hiramatsu e Akasaki).

73 Institute of Physics: Conferences Series 129, p. 851, 1991 (Akasaki, Amano, K. Itoh, N. Koide e K. Manabe).

74 Japanese Journal of Applied Physics, v. 34, p. L1517, 1995 (Akasaki, Amano, Shigetoshi Sota, Hiromitsu Sakai, Toshiyuki Tanaka e Masayoshi Koike).

75 Applied Physics Letters, v. 68, p. 2849, 1996 (Jinwook Burm, William J. Schaff, Lester F. Eastman, Amano e Akasaki).

76 Japanese Journal of Applied Physics, v. 36, p. L382, 1997 (Tetsuya Takeuchi, Sota, M. Katsuragawa, M. Komori, H. Takeuchi, Amano e Akasaki).

77 Journal of Applied Physics, v. 85, p. 7682, 1999 (Shigeo Yamaguchi, Michihiko Kariya, Shugo Nitta, T. Takeuchi, Christian Wetzel, Amano e Akasaki).

78 Applied Physics Letters, v. 76, p. 876, 2000 (Yamaguchi, Kariya, Nitta, T. Takeuchi, Wetzel, Amano e Akasaki).

79 Japanese Journal of Applied Physics, v. 39, p. 413, 2000 (T. Takeuchi, Amano e Akasaki).

${ }^{80}$ Applied Physics Letters, v. 89, p. 7820, 2001 (Yamaguchi, Kariya, Masayoshi Kosaki, Yohei Yukawa, Nitta, Amano e Akasaki).

81 Applied Physics Letters, v. 80, p. 802, 2002 (Yamaguchi, Yasuo Iwamura, Yasuhiro Watanabe, Kosaki, Yukawa, Nitta, Satoshi Kamiyama, Amano e Akasaki).

82 Applied Physics Letters, v. 105, a.n. 072101, 2014 (Daisuke Lida, Syunsuke Kawai, Nobuaki Ema, Takayoshi Tsuchiya, Motoaki Iwaya, T. Takeuchi, Kamiyama e Akasaki). 
and Realization of P-Type GaN by Mg-Doping Followed by LEEBI Treatment (08/12/2014, Stockholm University), bem como seus perfis no wikipedia (op. cit.).

Por fim, vejamos os trabalhos de Nakamura. Conforme destacamos no início deste artigo, depois que Nakamura obteve seu Mestrado em Engenharia Eletrônica, em 1979, na Universidade de Tokushima (UT) (note-se que, em 1994, ele recebeu o Doutorado em Engenharia ainda UT), foi trabalhar na Nichia Chemical Corporation, localizada também em Tokushima, na qual começou a pesquisar com o GaN. Nessa indústria, Nakamura desenvolveu um método similar ao de Amano e Akasaki, onde o A $\mathrm{N}$ foi substituído por uma fina camada de $\mathrm{GaN}$ e crescida em baixa temperatura. Esse trabalho foi apresentado, em 1991, por Nakamura $^{83}$; e por Nakamura, Masahuki Senoh e Takashi Mukai ${ }^{84}$. Ainda em $1991^{85}$, Nakamura, Yasuhiro Harada e Senoh utilizaram o MOVPE para crescer o GaN. Logo em 1992, a técnica do tunelamento de elétrons (EF-N), com a qual aceitadores de $\mathrm{Mg}$ e $\mathrm{Zn}$ formam estruturas complexas com o $\mathrm{H}$ servindo como neutralizante de dopantes, foi explicada por Nakamura, Naruhito Iwasa, Senoh e Mukai ${ }^{86}$ e por Nakamura, Mukai, Senoh e Iwasa ${ }^{87}$. Note-se que o efeito neutralizante do H já havia sido utilizado por Pankove, D. E. Carlson, Berkeyheiser e R. O. Wance, em 198388, e por G. F. Neumark Rothschild, em 1988 (US Patent 5252499).

Embora a Nichia tenha passado por dificuldades financeiras, Nakamura continuou a realizar suas pesquisas com HeS, visando a construção do LED azul-brilhante, usando ligas de AlGaN e InGaN para obter doador tipo-p como foi apresentado, em 199289, por Nakamura e Mukai; em 199390; e em 199491. Ainda na Nichia, em 199592, Nakamura apresentou seu famoso LED azul-brilhante com as HeS (InGaN/AlGaN) e, também em 199593, ele, Senoh, Iwasa e Nagahama construíram um LED azul-verde com a HeS de GaN, com uma eficiência

\footnotetext{
83 Japanese Journal of Applied Physics, v. 30, p. L1705, 1991.

84 Japanese Journal of Applied Physics, v. 39, p. 413, 1991.

85 Applied Physics Letters, v. 58, p. 2021, 1991.

86 Japanese Journal of Applied Physics, v. 31, p. 1258, 1992.

87 Japanese Journal of Applied Physics, v. 31, p. L138, 1992.

88 Physical Review Letters, v. 51, p. 2224, 1983.

89 Japanese Journal of Applied Physics, v. 31, p. L1457, 1992.

90 Japanese Journal of Applied Physics, v. 32, p. L8, 1993; Applied of Physics Letters, v. 62, p. 2390, 1993 (Nakamura, Senoh e Mukai); Journal of Applied Physics, v. 74, p. 3911, 1993 (Nakamura, Mukai, Senoh, Shin-ichi Nagahama e Iwasa).

91 Applied of Physics Letters, v. 64, p. 1687, 1994; Journal of Applied Physics, v. 76, p. 8189, 1994) (Nakamura, Mukai e Senoh).

92 Journal of Vacuum Science \& Technology A13, p. 105, 1995.

93 Japanese Journal of Applied Physics, v. 34, p. L797, 1995.
} 
acima de $10 \%$. Até deixar a Nichia, o que ocorreu em 1999, Nakamura e seu grupo investigou o ganho ótico de LED de InGaN, em 199694; em 199795; e em $1998^{96}$.

Destaque-se que, em 1997, Nakamura e Gerard Fasol publicaram o livro: Th Blue Laser Diode: GaN Light Emitters and Lasers (Springer-Verlag) e, em 2000, Nakamura, Fasol e Stephen J. Pearton publicaram o livro: The Blue Laser Diode: The Complete Story (Springer-Verlag).

Para detalhes dos trabalhos de Nakamura, ver sua Nobel Lecture: Shuji Nakamura, Background Story of the Invention of Efficient Blue InGaN Light Emitting Diode (08/12/2014, Stockholm University), bem como seu perfil no wikipedia (op. cit.).

94 Applied Physics Letters, v. 69, p. 1568; 3034; 4056, 1996 (Nakamura, Senoh, Nagahama, Iwasa, Takao Yamada, Toshio Matsushita, Yasunobu Sugimoto e Hiroyuki Kiyoku).

95 Applied Physics Letters, v. 70, p. 868; 1417, 1997 (Nakamura, Senoh, Nagahama, Iwasa, Yamada, Matsushita, Sugimoto e Kiyoku).

96 Applied Physics Letters, v. 72, p. 2014, 1998 (Nakamura, Senoh, Nagahama, Iwasa, Yamada, Matsushita, Kiyoku, Sugimoto, Tokuya Kozaki, Hitoshi Umemoto, Masahiko Sano e Kazuhuki Chocho). 